\title{
Obesity-induced hypermethylation of adiponectin gene
}

The mechanisms underlying obesityinduced dysregulation of the gene that encodes adiponectin (ADIPOQ) have been revealed in a new paper published in Nature Communications.

Adiponectin controls the metabolism of glucose and insulin, and its levels are decreased in obesity. However, the mechanism by which expression of $A D I P O Q$ is reduced in obesity is unknown. Jae Bum Kim and colleagues speculated that epigenetic changes could be involved in the suppression of ADIPOQ expression in obesity.

The researchers used bisulphite sequencing to analyse levels of DNA methylation at the adiponectin promoter in adipocytes isolated from mice fed either a normal chow diet or a high-fat diet (HFD). HFD-fed obese mice had more DNA methylation at the $\mathrm{R} 2$ region of the promoter than the lean mice fed a normal chow diet. Furthermore, the amount of methylation at the $\mathrm{R} 2$ region inverselycorrelated with levels of adiponectin mRNA.
Kim and colleagues were also able to demonstrate that levels of DNA methyltransferase 1 (DNMT1) were higher in the HFD-fed mice than in the lean mice. DNMT1 selectively methylated the $\mathrm{R} 2$ region, and also caused chromatin remodelling of the adiponectin promoter. Treating obese mice with a DNMT1 inhibitor (RG108) reduced methylation levels at the $\mathrm{R} 2$ region and increased levels of adiponectin.

"We have shown that adiponectin gene expression is dynamically regulated by epigenetic modifications, including DNA hypermethylation and chromatin remodelling at the adiponectin promoter, which could explain the decreased adiponectin expression in obese adipocytes," says Kim. The researchers hope that future studies examining the molecular mechanisms that control the selectivity of DNMT1 for its target site could aid development of therapies for obesity and its complications.

Claire Greenhill
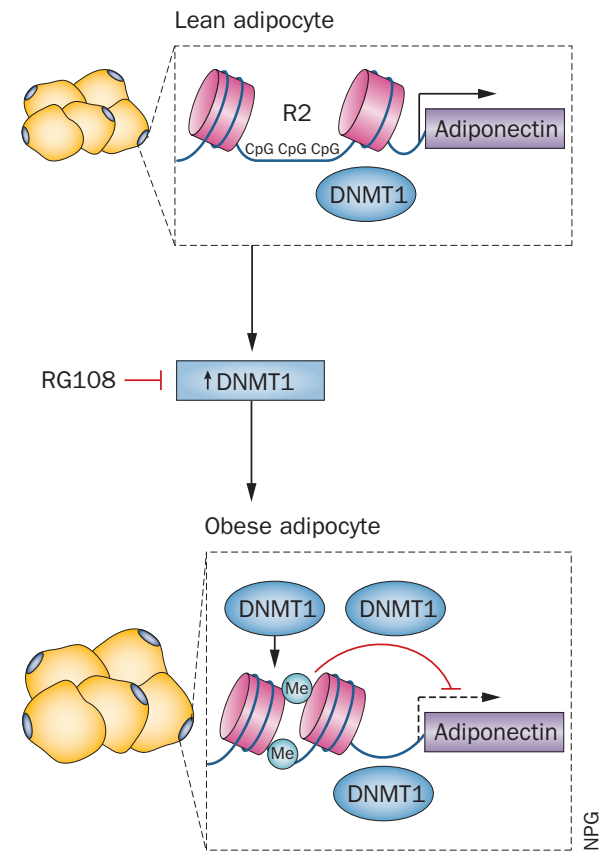

Original article Kim, A. Y. et al. Obesity-induced DNA hypermethylation of the adiponectin gene mediates insulin resistance. Nat. Commun. doi:10.1038/ncomms8585 